\title{
Fractional Dynamics
}

\author{
Carlo Cattani ${ }^{1, *}$ (i) and Renato Spigler ${ }^{2}$ \\ 1 Engineering School, DEIM, University of Tuscia, 01100 Viterbo, Italy \\ 2 Department of Mathematics and Physics, Roma Tre University, 00146 Rome, Italy; spigler@mat.uniroma3.it \\ * Correspondence: cattani@unitus.it
}

Received: 5 June 2018; Accepted: 11 June 2018; Published: 17 June 2018

Modelling, simulation, and applications of Fractional Calculus have recently become increasingly popular subjects, with impressive growth concerning applications. The founding and limited ideas on fractional derivatives have achieved an incredibly valuable status. The variety of applications in mathematics, physics, engineering, economics, biology, and medicine, have opened new, challenging fields of research. For instance, in soil mechanics, a suitable definition of the fractional operator has shed some light on viscoelasticity, explaining memory effects on materials. Needless to say, these applications require the development of practical mathematical tools in order to extract quantitative information from models, newly reformulated in terms of fractional differential equations. Even confining ourselves to the field of ordinary differential equations, the well-known Bagley-Torvik model showed that fractional derivatives may actually arise naturally within certain physical models, and are not merely fanciful mathematical generalizations. This Special Issue focuses on the most recent advances in fractional calculus, applied to dynamic problems, linear and nonlinear fractional ordinary and partial differential equations, integral fractional differential equations, and stochastic integral problems arising in all fields of science, engineering, and other applied fields.

In this issue, we have collected several significant papers devoted to applications of fractional methods with a focus on dynamical aspects. The applications range from theoretical mathematical-numerical aspects [1,2] to bio-medical subjects [3-7]. Applications to complex materials are investigated in [8], aiming at proposing a generalized definition of fractional operators. Special diffusion models are studied in [9-11].

\section{Numerical Aspects in Fractional Dynamics}

In recent years, many theoretical models have been used to investigate the main features of fractional calculus and the existence of links with the integer order operators. Although it seems quite natural to extend classical operators and models to fractional order operators, there are several unsolved problems related to such a non-trivial generalization. For instance, in [1], the authors show that the Gaussian provides the umbral image of Bessel and circular functions. In particular, they are able to single out the role played by spherical Bessel functions, and by a family of associated auxiliary polynomials, as transition elements between these families of functions.

Some nonlocal (fractional) operators in the Heisenberg group are given in [2]. Specifically, starting from the Grünwald-Letnikov derivative and the Marchaud derivative in a Euclidean setting, the author defines some nonlocal operators in the non-commutative structure of the first Heisenberg group, adapting the approach applied in the Euclidean case to this new framework.

\section{Fractional Methods in Bio-Medical Areas}

Applications of ordinary and fractional calculus to competition models in biology to bio-medical models based on suitable generalization of classical fluid-dynamics and diffusion have recently attracted the interest of several authors. In [3], the authors derive a fractional-order infectivity and recovery susceptible infectious recovered (SIR) model from the stochastic process of a continuous time random walk that incorporates a time-since-infection dependence on both the infectivity and the 
recovery of the population. By considering a power-law dependence between infectivity and recovery, the authors show that fractional-order derivatives appear in generalized master equations that govern the evolution of SIR populations. Moreover, the Authors are able to show that, under appropriate limits, this fractional-order infectivity and recovery model reduces to both the standard SIR model and the fractional recovery SIR model.

In [4], the authors propose a fractional complex permittivity model of dielectric media with memory. In particular, they show that the fractional order depends on the frequency band of excitation energy, according to the second Principle of Thermodynamics. It is an interesting fact that this model is indeed validated by measurements actually made on biological tissues.

In [5], a Mathematical model has been proposed based on the Caputo fractional derivative, to analyze cerebral microaneurysms. In this paper, the author proposes a model to describe the formation of microaneurysms that involves the chemo-mechanical coupling of blood and endothelial and neuroglial cells. It shows how to model blood as a non-local non-Newtonian incompressible fluid, and to solve analytically the Poiseuille flow of such a fluid through an axisymmetric circular rigid and impermeable pipe in the presence of wall slip. The spatial derivatives of the proposed generalization of the rate of deformation tensor are expressed using Caputo fractional derivatives. The wall slip is represented by the classic Navier law and a generalization of this law involving fractional derivatives. By numerical simulations, it is also shown that, as expected, hypertension is one of the major reasons for microaneurysmal formation.

A collocation method based on fractional B-splines for the solution of fractional differential problems was developed in [6]. The key-idea proposed by the author is to use the space generated by the fractional B-splines, i.e., piecewise polynomials of non-integer degree, as approximating space. Then, in the collocation step, the fractional derivative of the approximating function is approximated accurately and efficiently by an exact differentiation rule that involves the generalized finite difference operator. This approach is particularly suitable for the numerical solution of nonlinear dynamical systems of fractional order, so that the Author shows an application by solving the fractional Lotka-Volterra model and the fractional predator-pray model with variable coefficients. In this paper, the author also analyzes the accuracy of the method, and in particular, shows that it is accurate and has a low computational cost.

In [7] the authors show that time fractional generalization of the cable equation arises naturally in the continuous time random walk, by considering a superposition of Markovian processes and in a ggBm-like construction of the random variable. This model is also used to describe tranmembrane potentital in spiny dendrites.

\section{Fractional Methods in Complex Materials}

The extension of fractional order derivatives to distributed order fractional derivatives is rather simple from a formal point of view, but it does not yet have a simple, obvious analytic form that allows its fast numerical computation. This is necessary when solving "differential equations" containing this kind of derivative. In [8], the authors provide a simple analytic kernel for the Caputo-distributed order fractional derivative and the Caputo-Fabrizio-distributed order fractional derivative, which may be used for numerical purposes in cases where the weight function is unity. With this new approach, a faster solution of fractional differential equations is easily obtained. In this paper, the author also proposes a model to describe composite materials that include different constituents, and shows its compatibility with thermodynamics.

\section{Fractional Dynamics in Diffusion Models}

One of the main problems in fractional calculus is to single out the role played by the fractional order of derivatives. In other words, in many recent papers on fractional calculus, the main struggle was to find a significant physical meaning of the fractional order. In [10], the authors made an attempt at identifying the orders of the fractional derivatives in a simple anomalous diffusion model, based 
on real data. By using a one-dimensional fractional diffusion equation, they are able to fit the data by choosing several values of the fractional orders and computing the infinite-norm "errors", representing the deviation between numerical solution and experimental data. The minimal error is obtained by a time fractional order equal to about 0.6 and a space fractional order of about 2 . These results accurately describe the memory properties of the porous medium that was experimentally observed.

In [9], the authors investigate the anomalous deviations from the Beer-Lambert law that has been observed in a wide range of applications. Despite attempts to explain this phenomenon, a reliable and accepted model has not been provided so far. In this paper, the authors consider deviations from the classical exponential extinction, introducing a weighted version of the classical law. By using this generalized law, they are able to show both sub- or super-exponential extinction of radiation. Moreover, this generalized law can be extended to the case of inhomogeneous media.

The dust aerosols floating in the atmosphere of Mars cause an attenuation of the solar radiation crossing the atmosphere that cannot be modelled through classical diffusion processes. In [11], the authors give the definition of a type of fractional diffusion equation which provides a more accurate model for such dynamics. The second order moment of this equation allows us to establish a connection between the fractional equation and the Ångstrom law that models the attenuation of solar radiation. Exploiting their model, the authors investigate three dimensional wavelength-fractional diffusion equations, and they obtain the analytical, as well as numerical, solutions using two different approaches of the fractional derivative.

Funding: This research received no external funding.

Conflicts of Interest: The authors declare no conflict of interest.

\section{References}

1. Dattoli, G.; di Palma, E.; Licciardi, S.; Sabia, E. From Circular to Bessel Functions: A Transition through the Umbral Method. Fractal Fract. 2017, 1, 9. [CrossRef]

2. Ferrari, F. Some Nonlocal Operators in the First Heisenberg Group. Fractal Fract. 2017, 1, 15. [CrossRef]

3. Angstmann, C.N.; Henry, B.I.; McGann, A.V. A Fractional-Order Infectivity and Recovery SIR Model. Fractal Fract. 2017, 1, 11. [CrossRef]

4. Ciancio, A.; Flora, B.F.F. A Fractional Complex Permittivity Model of Media with Dielectric Relaxation. Fractal Fract. 2017, 1, 4. [CrossRef]

5. Drapaca, C.S. Poiseuille Flow of a Non-Local Non-Newtonian Fluid with Wall Slip: A First Step in Modeling Cerebral Microaneurysms. Fractal Fract. 2018, 2, 9. [CrossRef]

6. Pitolli, F. A Fractional B-spline Collocation Method for the Numerical Solution of Fractional Predator-Prey Models. Fractal Fract. 2018, 2, 13. [CrossRef]

7. Vitali, S.; Mainardi, F.; Castellani, G. Emergence of Fractional Kinetics in Spiny Dendrites. Fractal Fract. 2018, 2, 6. [CrossRef]

8. Caputo, M.; Fabrizio, M. The Kernel of the Distributed Order Fractional Derivatives with an Application to Complex Materials. Fractal Fract. 2017, 1, 13. [CrossRef]

9. Casasanta, G.; Garra, R. Towards a Generalized Beer-Lambert Law. Fractal Fract. 2018, 2, 8. [CrossRef]

10. Concezzi, M.; Spigler, R. Identifying the Fractional Orders in Anomalous Diffusion Models from Real Data. Fractal Fract. 2018, 2, 14. [CrossRef]

11. Jimenez, S.; Usero, D.; Vasquez, L.; Velasco, M.P. Fractional Diffusion Models for the Atmosphere of Mars. Fractal Fract. 2018, 2, 1. [CrossRef] 